\title{
Relaparotomy after Cesarean Section in a Tertiary Center
}

\author{
Sandesh Poudel ${ }^{1}$, Shree Prasad Adhikari ${ }^{1}$, Snigdha Rai ${ }^{1}$, Sangeeta Mishra ${ }^{1}$, Renuka Tamrakar ${ }^{2}$
}

\section{Author Info:}

'Paropakar Maternity and Women's Hospital, Thapathali, Kathmandu

${ }^{2}$ Assistant Professor, Chitwan Medical College Teaching Hospital, Bharatpur, Chitwan

\section{Corresponding Author:}

Dr. Sandesh Poudel

Contact/Email:

sandeshjyotipoudel@gmail.com

+9779851064246

ORCID iD: https://orcid. org/0000-0002-6852-1211

\begin{abstract}
Background: Relaparotomy after cesarean section is associated with significant maternal morbidity and mortality which can be prevented with proper precaution during the primary surgery. The objective of the study was to determine factors associated with relaparotomy after cesarean section.
\end{abstract}

Methods: A hospital based descriptive cross sectional study conducted by analyzing the records of 3 years from 1st July 2017 to 30th June 2020, among the women who underwent relaparotomy after cesarean section at Paropakar Maternity and Women's Hospital, Kathmandu.

Results: There were 21,270 cesarean sections among 64,475 deliveries with the mean incidence of $32.6 \%$. A total of 22 patients underwent relaparotomy out of which 18 had their primary surgery performed at our hospital i.e. $0.08 \%$ of the total caesarean section, and 4 cases were referred from peripheries. $60 \%$ of primary surgery were done by residents and the commonest indications of primary surgery were previous CS (36.3\%) and obstructed labor (27.2\%). Primary PPH was the major indication for laparotomy (31.8\%) with mean interval from primary surgery of 4.7 hours followed by burst abdomen (22.7\%) and secondary PPH (18.1\%). Hysterectomy was the commonest operative intervention done during relaparotomy accounting for $45.4 \%$ followed by tension suture for burst abdomen (18\%). There were 2 maternal death among them which were due to aspiration pneumonia following eclapmsia and DIC.

Conclusion: Relaparotomy has increased risk of adverse maternal outcome but still is a lifesaving intervention. Early recognition, timely intervention and multidisciplinary teamwork helps to decrease the complications associated with it.

Keywords: cesarean section, CS, relaparotomy, morbidity

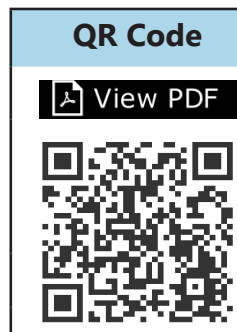

Scan Me

\section{Article Info}

Received: 1 December 2020; $\quad$ Accepted: : 24 January 2021;

Published Online: 25 January 2021

How to cite this article in Vancouver Style?

Poudel S, Adhikari SP, Rai S, Mishra S, Tamrakar R. Relaparotomy after Cesarean Section in a Tertiary Center. Europasian J Med Sci. 2021;3(1):6-11. https://doi.org/10.46405/ejms.v3i1.287

\section{Disclaimer}

Conflict of Interest: None Declared;

Source of Support: Nil

Copyright (C) 2021 by author(s). This work is licensed under the terms and condition of Creative Commons Attribution International License 4.0@ (1) (http://creativecommons.org/licenses/by/4.0/) which permits unrestricted use, distribution, and reproduction in any medium, provided the original work is properly cited. This is an open access publication, and can be downloaded freely from the website of the Journal: www.europasianjournals.org. The Journal as well as publisher remain neutral with regards to any jurisdictional claims in any published articles, its contents and the institutional affiliations of the authors. The Europasian Journal of Medical Sciences (EJMS) (www.europasianjournals.org) is an official Journal of Nirvana Psychosocial Care Center \& Ressearch Institute (www.nirvanapscc. com). 


\section{INTRODUCTION}

Cesarean sections are effective in saving maternal and infant lives, but only when they are required for obstetrically indicated reasons. At a population level, cesarean section rates higher than $10 \%$ are not associated with reductions in maternal and newborn mortality rates. ${ }^{1}$ The risk of complications especially mortality after Cesarean section is five to seven times higher than the vaginal deliveries. ${ }^{2,3}$

One of the most serious and fearful incident following any obstetric abdominal surgery like the cesarean section in the post-operative period is relaparotomy, which is a life threatening possibility. Relaparotomy refers to operations performed within 60 days in association with the initial surgery. ${ }^{4}$ Relaparotomy poses not only a crucial challenge for the patient to undergo repeat surgery within a short period of time but also a big dilemma for the obstetrician regarding the decision and the timing to perform re-laparotomy. Post relaparotomy complications and ICU admissions are equally stressful to both the patients and surgeons because of associated significant morbidity, mortality, and economic burden. There has been a recent increase in primary cesarean sections for the last two decades due to changes in maternal characteristics, increased maternal and fetal safety concerns among many others. ${ }^{5}$ With the increasing rate of cesarean sections, complications related to them are increasing and so are the relaparotomies.

The major indications of relaparotomy are primary and secondary $\mathrm{PPH}$, burst abdomen, slippage of ligature, rectus sheath hematoma and infections due to leak. ${ }^{6,7,8}$ Due to significant morbidity and mortality associated with re-laparotomies, one should be very vigilant on measures during primary surgery to avoid it. Measures like proper pre-operative workup, proper light and exposure, newer antibiotics and proper antiseptics, better post-operative fluid and electrolyte balance, proper surgical techniques, secured hemostasis, complete exploration and appropriate drainage are the few among many which can reduce relaparotomies. ${ }^{9}$ This study will help to determine the factors and risk situations that are associated with relaparotomy in obstetrics practice so that they can be worked upon by all concerned departments as well as aid the obstetrician to be more vigilant and be cautious in the primary surgery to prevent the further secondary surgical intervention and its morbidities.

\section{MATERIALS AND METHODS}

This was a descriptive cross-sectional study done over the period of 3 years, 1st July 2017 to 30th June 2020, in Paropakar Maternity and Women's Hospital which is the largest tertiary care referral hospital in Nepal and a major post graduate teaching institute. It receives referrals from almost all parts of the country by road and with airlift service provided by the government free of cost to those emergency cases which cannot be managed in districts. Cesarean sections are performed by all the duty doctors including trainees, registrars, senior registrars and consultants depending upon the indication of primary surgery following all the protocols for preoperative, intraoperative and postoperative care.

All the cases that have undergone relaparotomy within 60 days for caesarean section including both in house and referred in, during the time period of 3 years have been included in the study. Prior approval for data collection was taken from Institutional Review Committee (IRC) with approval reference number of $60 / 1661$. All the cases were identified from the records of operation theatre and MICU. Inpatient charts and medical records of all the cases were retrieved from the Record Section. The data collected were then entered into the excel sheet according to the age, parity, indications for $\mathrm{CS}$, indications of relaparotomy, the time interval between completion of the CS and reopening of the abdomen and the type of interventions done. Level of principal surgeon involved during both cesarean section and relaparotomy were noted. Further data on requirement of the blood transfusion, duration of MICU stay and final outcome including mortality were analyzed and finally depicted in the form of tables and diagrams.

\section{RESULTS}

During the study period of three years, there were 21,270 cesarean sections among 64,475 deliveries. The cesarean section rate rose from $29.4 \%$ in the first year to $35.4 \%$ in the third year of the study with the mean incidence of $32.6 \%$. A total of 22 patients underwent relaparotomy after cesarean section out of which $81.8 \%$ of primary surgeries were emergency. Among the relaparotomy cases, 18 had their primary surgery performed at our hospital i.e. $0.08 \%$ of the total caesarean section, and 4 cases were referred from peripheries. $90 \%$ of the relaparotomy cases needed ICU admission and 1 among 5 burst abdomen was referred to multispecialty center after surgery for further 


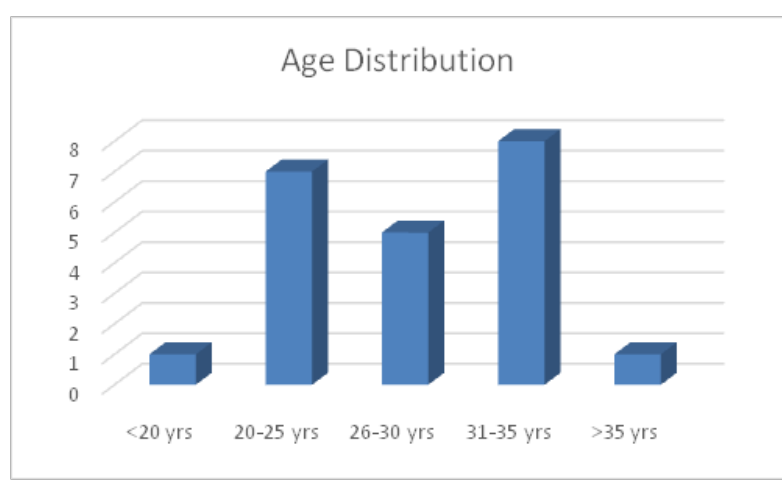

Figure 1: Age distribution of the participants

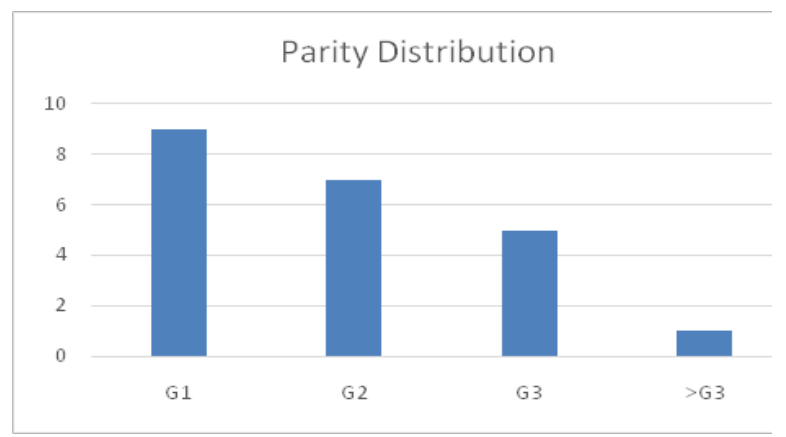

Figure 2: Parity-wise distribution of the participants

Table 1: Indications of Primary Cesarean Section Indications of primary CS

Previous CS

Obstructed labor including DTA

Institution $(n=18)$

Fetal distress

Severe Preeclampsia/ Eclampsia

Placenta previa

Hand prolapse relaparotomies, maximum number of primary CS done in our hospital were by the trainee residents (60\%) followed by senior house officers and three cases were done by consultants while all the relaparotomy were done by consultants.

Among the patients who underwent relaparotomy after cesarean section, for most of the patients i.e. $36.3 \%$, the indication for CS was previous CS, followed by obstructed labour (27.2\%), fetal distress (13.6\%), hypertensive disorder (9\%), placenta previa (9\%) and hand prolapse (4.5\%) as shown in Table 1. One relaparotomy was done in a patient who had undergone peripartum hysterectomy for massive PPH during CS for placenta previa while 2 had chorioamnionitis and preexisting anaemia.

The relation between indications for relaparotomy and time interval from primary surgery is analyzed in Table 2. Primary PPH and burst abdomen due to wound dehiscence were the commonest indications for the relaparotomy with incidence of $31.8 \%$ and $22.7 \%$ respectively. All cases of primary PPH were due to atony with the mean time interval of 4.7 hours from the time of primary surgery. Of the 5 burst abdomen one had a gangrenous jejunal loop which was managed with the help of gastro surgeon

$\begin{array}{cl}\text { Referred in }(n=4) & \text { Total }(n=22) \\ - & 8(36.3 \%) \\ 1 & 6(27.2 \%) \\ 1 & 3(13.6 \%) \\ 1 & 2(9 \%) \\ - & 2(9 \%) \\ 1 & 1(4.5 \%)\end{array}$

Table 2: Indications of relaparotomy and time interval from primary surgery

Indications of relaparotomy

Primary PPH

Burst Abdomen

Secondary PPH

Hemoperitoneum

Rectus Sheath Hematoma

Lower uterine segment hematoma
Time Interval ( mean)

30 min-10 hours (mean 4.7 hours)

8-26 days (mean 13.4 days)

14-22 days (mean 17.2 days)

5-23 hours (mean 12 hours)

2-5 days (3.5 days)

8 hours
Number $(\mathrm{n}=22)$

7 (31.8\%)

$5(22.7 \%)$

$4(18.1 \%)$

$3(13.6 \%)$

$2(9 \%)$

$1(4.5 \%)$ surgical management. There were 2 mortalities due to aspiration pneumonia following eclapmsia and DIC. Sociodemographic profile according to age and parity of the patients who underwent relaparotomy after cesarean section is shown in Figure 1 and 2. Majority of the patients were between the age group of $31-35$ years (36\%) and least below 20 years of age (5\%) while $41 \%$ of the patients were primigravida. Among all the and others were related to infections. $18.1 \%$ of relaparotomy cases were done for secondary $\mathrm{PPH}$ with mean time interval of 17 days from primary surgery. There were 3 relaparotomies for postoperative hemoperitoneum which occurred due to bleeding from the vault, uterine vessels and tubal vessels slipped following bilateral tubal ligation.

Total and subtotal hysterectomy accounted for major interventions performed during re- 
Table 3: Intraoperative Interventions

Procedure done during relaparotomy Number Total hysterectomy

$5(22.7 \%)$

Subtotal hysterectomy

$5(22.7 \%)$

Debridement with tension suture

$4(18.1 \%)$

Hemostatic suture/Compression suture3 (13.6\%)

Drainage of hematoma

$2(9 \%)$

Small bowel Resection Anastomosis with tension suture

$1(4.5 \%)$

Repair of uterine dehiscence

$1(4.5 \%)$

B-Lynch Suture with Bilateral uterine artery liga-

tion

$1(4.5 \%)$

laparotomy accounting for $45.4 \%$ of total interventions as depicted in Table 3 which included 6 for primary $\mathrm{PPH}$ and 3 for secondary PPH. One case of secondary hemorrhage where there was uterine dehiscence was managed with repair of uterine layer after debridement of unhealthy tissues while one patient with re-laparotomy done for primary $\mathrm{PPH}$ underwent with bilateral uterine artery ligation along with B Lynch compression suture. All the patients with primary and secondary hemorrhage were transfused with blood products during intra and postoperative periods. Out of 5 burst abdomen cases, 4 of them were managed with tension suture applied over the rectus with number 1 non absorbable suture while one underwent small bowel resection and anastomoses before application of tension suture.

\section{DISCUSSION}

Paropakar Maternity and Women's Hospital is the largest maternity care center in the country with referrals of complicated cases from all over the country as well. The cesarean section rate of $32.6 \%$ in seen in present study is comparable with Bakirkoy Maternity and Children Diseases Hospital in Turkey with a mean CS rate of $31.23 \%$ as shown in a study by Gedikbasi et al. ${ }^{7}$ They encountered 35 re-laparotomy cases in 5 year with incidence of $0.12 \%$ while the present study revealed incidence of $0.08 \%$ excluding 4 relaparotomy that have been referred to us from outside our institution. Similarly an incidence of $0.16 \%$ of relaparotomy was reported in a government hospital of India who had CS rate of $60.28 \%$ as shown by Rather et al. ${ }^{10}$ Hadar et al. ${ }^{11}$ reported higher rates of postoperative complications when cesarean sections are done by residents which is similar to our study where $60 \%$ the primary cesarean section who underwent relaparotomy were done by residents.

Emergency indications for cesarean section was the major contributing factor for relaparotmy i.e.
$81.8 \%$ which is in line with most of the previous studies. ${ }^{4,6,7,8}$ Surgeries performed urgently and emergently had higher rates of morbidity and mortality compared to elective ones as shown in a study by Mullen et al. which highlighted the need for improved risk stratification. ${ }^{12}$ Lack of proper preoperative checkup, urgent need of delivery, changes in anatomy of the lower segment of uterus and bladder associated during advanced labor, lack of availability of adequate set of manpower and increased chances of sepsis are probably common causes of morbidity and mortality associated with emergency procedure.

Our study showed previous cesarean section and obstructed labor were the major indications of primary surgery, $36.3 \%$ and $27.2 \%$ respectively which is similar to the study done by Kumari $A$ and Vidyarthi A who found previous CS and obstructed labor as primary indications of cesarean section among patients who underwent relaparotomy (21.4 and 32.14\%). ${ }^{13}$ Also Gedikbasi et al have similar findings with $29 \%$ of the relaparotomy in the patients with previous cesarean sections. ${ }^{7}$ Complications like relaparotomy increases in previous sections due to risk of dense adhesions, associated placental abnormality and difficulty in securing hemostasis. Similarly increased associated trauma to lower uterine segment, uterine arteries, cervix, bladder and vagina, hemorrhage and sepsis in cesarean sections done for obstructed labor including deep transverse arrest can predispose to relaparotomy.

Hemorrhage was the leading cause of relaparotomy after cesarean section with occurrence of $70 \%$ followed by burst abdomen as reported in a study done in Israel by Kasseous et al. ${ }^{14}$ Raagab et al. ${ }^{15}$ has also stated hemorrhage including both intraabdominal bleeding and uncontrolled PPH as the primary cause (92.3\%). These study results are comparable to ours which showed hemorrhage as the commonest indication of relaparotomy (64.5\%) including primary $\mathrm{PPH}$, secondary $\mathrm{PPH}$ and hemoperitoneum. Most of interventions during repalarotomy in the present study were either total or subtotal hysterectomy, after failed uterine saving procedures (45.4\%) like stepwise devascularisation. Similar findings were observed by Seffa JD with $38.6 \%$ of relaparotomy after cesarean section underwent hysterectomy as a lifesaving procedure. ${ }^{16}$ Secondary PPH following CS is often difficult to control by vessel ligation, hence requires hysterectomy in most of the cases. Proper hemostasis during the CS with prophylactic use 
of antibiotics may well reduce infection and thus chances of secondary PPH. ${ }^{17}$

Post cesarean surgical site infection is the likely risk factor for burst abdomen in our study. Out of five burst abdomen two cases had Staphylococcus aureus in their culture report while one had $E$. coli and all the primary surgeries who had wound dehiscence were emergency surgeries where risk of infection is high. Similar results were seen when a study in Pakistan, assessed risk factors of wound dehiscence and found that emergency surgery, bowel injury and surgical site infection were the commonest cause beside malignancy and age factor. ${ }^{18}$

With two mortalities in the present study due to DIC and aspiration pneumonia following eclampsia, case fatality rate of relaparotomy after cesarean section was $9 \%$. Similar mortality rate of $9 \%$ and $12 \%$ has been reported by Seffa et al. ${ }^{14}$ and Seal et al. ${ }^{6}$ respectively while the higher rate of $25 \%$ rate was discovered by Elkhateeb et al. ${ }^{19}$ from Egypt with all three studies reporting mortality due to sepsis, hemorrhage and organ failure. Early recognition, timely intervention and multidisciplinary team approach are the milestones to prevent mortality associated with relaparotomy.

\section{CONCLUSION}

Relaparomy is a lifesaving intervention which is even more relevant in current situation where CS section rates are increasing. Primary $\mathrm{PPH}$, secondary $\mathrm{PPH}$ and wound dehiscence are the common indications for relaparotomy associated with significant maternal morbidity and mortality. Early recognition, timely intervention with experienced obstetrician and multidisciplinary teamwork helps to decrease the complications associated with it.

\section{REFERENCES}

1. World Health Organization. WHO statement on Cesarean section rates. 2015 https://apps. who.int/iris/bitstream/handle/10665/161442/ WHO_RHR_15.02_eng.pdf?sequence $=1$

2. Danforth DN. Cesarean section. JAMA. 1985; 253(6):811-18. https://doi:10.1001/ jama.1985.03350300099029

3. Schuitemaker $\mathrm{N}$, van Roosmalen J, Dekker $G$, van Dongen $P$, van Geijn H, Gravenhorst JB. Maternal mortality after cesarean section in The Netherlands. Acta Obstet Gynecol Scand. 1997; 76 (4):332-4. https://doi. org/10.1111/j.1600-0412.1997.tb07987.x

4. Unalp HR, Kamer E, Kar H, Bal A, Peskersoy M, Ali Onal M. Urgent abdominal re-explorations. World J Emerg Surg. 2006; 1:10. https://doi. org/10.1186/1749-7922-1-10

5. Joseph KS, Young DC, Dodds L, O'Connell CM, Allen VM, Chandra S et al. Changes in maternal characteristics and obstetric practice and recent increases in primary cesarean delivery. Obstet Gynecol. 2003; 102(4):791-800. https:// doi.org/10.1016/s0029-7844(03)00620-3

6. Seal SL, Kamilya G, Bhattacharyya SK, Mukherji J, Bhattacharya AR. Relaparotomy after cesarean delivery: experience from an Indian teaching hospital. J Obstet Gynaecol Res. 2007; 33(6):804-9. https://doi.org/10.1111/j.14470756.2007.00660.x

7. Gedikbasi A, Akyol A, Asar E, Bingol B, Uncu $R$, Sargin A et al. Re-laparotomy after cesarean section: operative complications in surgical delivery. Arch Gynecol Obstet. 2008; 278(5):41925. https://doi.org/10.1007/s00404-008-06049

8. Shrestha R, Karki L, Shrestha S, Malla A, Pradhan $B$, Singh A et al. Relaparotomy after caesarean section. J Patan Academy Health Scien. 2016; 3(2), 28-31.

9. Patel H, Patel $P$, Shah DK. Relaparotomy in general surgery department of tertiary care hospital of Western India. Int Surg J. 2016; 4(1):344-7. https://doi.org/10.18203/23492902.isj20164467

10. Rather SY, Sultana S, Shaheena, Anim ZA, Alam I. Indications for Relaparotomy in Cesarean Section in a Tertiary Care Hospital of a Developing Country. Int J Scien Res Pub. 2015;5(10)

11. Hadar E, Melamed N, Tzadikevitch-Geffen K, Yogev $\mathrm{Y}$. Timing and risk factors of maternal complications of cesarean section. Arch Gynecol Obstet. 2011;283(4):735-41. https:// doi.org/10.1007/s00404-010-1450-0

12. Mullen MG, Michaels AD, Mehaffey JH, Guidry CA, Turrentine FE, Hedrick TL. Risk associated with Complications and Mortality after Urgent surgery vs Elective and Emergency surgery. JAMA Surg. 2017;152(8):768-74. doi: 10.1001/ jamasurg.2017.0918

13. Kumara A, Vidhyarthi A. An analysis of relaparotomy in operative obstetrics. Int J 
Reprod Contracept Obstet Gynecol 2020; 9(5):1813-19 https://doi.org/10.18203/23201770.ijrcog20201560

14. Kessous $R$, Danor $D$, Weintraub $Y A$, Wiznitzer A, Sergienko R, Ohel I et al. Risk factors for relaparotomy after cesarean section. J Matern Fetal Neonatal Med. 2012; 25(11):2167-70. https://doi.org/ $10.3109 / 14767058.2012 .668978$

15. Raagab AE, Mesbah $\mathrm{YH}$, Brakat Rl, Zayed AA, Alsaammani MA. Re-laparotomy After Cesarean Section: Risk, Indications and Management Options. Med Arh. 2014; 68(1): 44-43. https:// doi.org/10.5455/medarh.2014.68.41-43

16. Seffah JD. Relaparotomy after cesarean section. Int J Gynecol Obstet.2005; 88(3):253-7. https:// doi.org/10.1016/j.ijgo.2004.12.014

17. Akther R, Hossain T, Rashid M. Relaparotomy after cesarean delivery: a prospective study. J Dhaka Med Coll. 2011; 20(1):57-62. https://doi. org/10.3329/jdmc.v20i1.8583

18. Khan MN, Naqvi AH, Irshad K, Chaudhary AR. Frequency and risk factor of abdominal wound dehiscence. J Coll Physicians Surg Pak. 2004; 14(6):355-7. https://doi.org/06.2004/ jcpsp.355357

19. Elkhateeb R, Mahran A, Sanad AS, Bahaa HA. Re-Laparotomy after Caesarean Section at a Tertiary Hospital in Egypt: Cross Sectional Study. Gynecol Obstet. 2017; 7: 1-4. https://doi. org/10.4172/2161-0932.1000433 